\title{
Photothermal investigations on advanced ceramics
}

\author{
by J. Geerkens ${ }^{1}$, B. Schmitz ${ }^{1}$ and G. Goch ${ }^{1,2}$ \\ 1 Institut für Lasertechnologien in der Medizin und Meßtechnik an der Universität UIm, Helmholtzstraße \\ 12, D-89081 Ulm, Germany. 2 Universität Ulm, Abteilung Meß-, Regel- und Mikrotechnik, Bereich \\ MeßtechnikAlbert-Einstein-Allee 41, D-89069 UIm, Germany
}

\begin{abstract}
Mechanical or thermal impacts on surfaces and near-surface layers during manufacturing processes often result in structural changes. They become detectable by photothermal techniques, when they correlate with alterations of thermal properties. We apply this approach on the detection of variations in ceramic surfaces. Vickers indentations model the mechanical load during grinding and a thermal surface treatment was performed by pulsed laser illumination. From the photothermal signals the depths and profiles of thermal conductivity variations depending on the different loading strengths could be reconstructed.
\end{abstract}

\section{Introduction}

During the past few years advanced ceramic materials gathered great importance in manufacturing technologies [1]. This is mainly due to their good performance concerning hardness, ductility and wear resistance yielding increased manufacturing speeds. With respect to their further applicability, reliablility and functionality advanced ceramics have to fulfill high demands on shape, accuracy and surface finishing. For most applications grinding with diamond wheels is the final step during the manufacturing process of ceramic tools. During this process the diamond grains can induce micro-cracks and plastic deformations in the surface region of the ceramics. This may lead to a higher possibility of a damage when the ceramic tools are in use. The idea to heal these micro-cracks by laser heating of the surface after the grinding process is under investigation by various groups.

The plastic deformation, resulting in micro-structural changes as well as the micro-cracks take influence on the thermal conductivity of the concerned region and therefore photothermal measurement techniques yield information about the thermal properties of the surface and near surface region [2]. To some extent mechanical or structural properties can be correlated with thermal ones which leads to an evaluation of technological properties like hardness etc.

In order to clarify the metrological problem, figure 1a demonstrates the change in surface layers during a mechanical impact while grinding or polishing a ceramic surface [1]. Due to the mechanical and thermal load brittle fracture, surface defects, cracks and subsurface damages can be induced by the material removal and increased material compressions. The impacts of local pressures and heat in the contact zones during the machining process cause plastic deformations. Since all these processes significantly influence the mechanical strength and affect the later use of ceramics, their effects on materials' properties have to be determined nondestructively and contactless. As these changes happen in near-surface layers and are probably combined with variations of the thermal properties, they can be detected by photothermal means.

Our special interest in this field is focused on the detection of altered zones caused by a manufacturing process and the thermal variations in near-surface layers due to mechanical or laser material processing. Basically, we assume that the varied mechanical properties, are caused by locally distributed inhomogeneities on and beneath the sample's surface (e.g. cracks, delaminations, alterations of microcristalline structures, varied material densifications, porosities, etc.). In order to model the alterations of thermal properties (which are particularly correlated with mechanical parameters) by a slight mechanical load, we chose the Vickers 
hardness check. According to figure $1 b$, this 'indentation fracture mechanics' approach [7] assumes that the damage produced by grinding can be modeled by a flaw system produced by a sharp indentor.

\section{Experimental configuration}

In a photothermal experiment, heat is generated by the interaction between an intensitymodulated light beam and an extended, three-dimensional sample. Absorption and subsequent deexcitation-relaxation release heat energy that may be distributed throughout a large region of the sample or be confined to a small region such as the surface of an opaque solid. The corresponding thermal response of the periodically heated solid can be detected by different sensing devices. In the Optical Beam Deflection (OBD) setup [8] the local thermal response of the periodically heated solid is detected by the deflection of a low-power probe laser beam while it crosses the air region adjacent to the heated surface spot in grazing incidence. The deflection implies information about the thermal material properties and the identification and distribution of subsurface hidden structures. In photothermal radiometry [9] (PTR), the variations of the surface temperature are monitored by an infrared sensitive device, e.g. a MCT-detector. For real samples, various properties affect the measured IR-flux (optical material properties, absolute temperature of the surrounding, surface roughness, heat flow from the sample's surface to the surrounding gas, etc.). Usually, these influences are ignored in nondestructive photothermal applications, since only the signal changes reveal the subsurface structures during the scanning procedures.

For our experiments (see figure 2) we use an argon laser for excitation. Either a mechanical chopper or an acousto-optic device modulates the laser light. After expansion the laser beam is tightly focused on the sample's surface. Thus, experiments with a resolution in a microscopic scale can be realized. In order to detect the photothermal deflection caused by the temperature gradient in a thin air layer above the heated spot (OBD), the commercially available compact setup 'Mirage MonoBloc' has been implemented. Within this unit a HeNe laser beam skimmes parallel to the sample's surface. One of the two deflection contributions (normal or transverse component) is measured by a four quadrant diode. In the radiometric setup the variations of the surface temperture are monitored by a Joule-Thomson cooled MCT sensor. The emitted IRradiation is imaged onto the detection plane by two $\mathrm{ZnSe}$ lenses. After preamplifying the different outputs are fed into the two lock-in amplifiers. The samples themselves are mounted on computer controlled $X-Y$ stages to moving them relative to the detectors and the laser beams. While scanning laterally across a buried nonunifomity inside the samples, the OBDand the radiometric signals are recording simultaneously spatial variations of the photothermal signals' amplitude and phase.

In terms of the photothermal results we express the detectability of deviations in the thermal properties by means of the amplitude and phase contrast functions [3]. These measurable values quantify the signal variations in the presence of a defect or thermal alteration compared to the background signal of the surrounding homogeneous material. In order to reconstruct the depth and profiles of thermal changes the contrast functions must be determined in dependence of the modulation frequency $f$.

\section{Survey of the theoretical photothermal description}

The theoretical basis for the identification of subsurface thermal inhomogenieties and for the reconstruction of thermal parameters $[3,4,6,10]$ is described in detail elsewhere. Therefore, a very brief overview will be outlined in the following.

The basic theoretical aspects use the fact that the penetration depth of the thermal waves can be controlled by the modulation frequency of the excitation. Consequently, the photothermal signals, which were registered from the surface only, carry still information on local thermal properties. Thus, the solutions of the heat diffusion equation (HDE) with space dependent coefficients have to be known in order to reconstruct the depth profiles by an 
inversion of the measured frequency sweeps. The amplitude of the temperature distribution describes the absolute temperature value while its argument corresponds to the phase shifts between the exciting light source and the temperature rise. Under certain restrictive conditions suitable analytical or numerical algorithms can be found: the sample must be opaque or vice versa, the optical penetration depth must be much smaller than the thermal diffusion length $\mu$ (given by the thermal parameters $\mathrm{k}$ : thermal conductivity, pc: specific heat capacity and $\omega$ : modulation frequency); only the thermal conductivity $k$ changes with depth while the heat capacity remains unchanged. The last assumption might be reasonable because microscopic cracks or internal interfaces vary the microscopic magnitude $k$ rather than the macroscopic property $\rho \mathrm{c}$. Further on, the condition for a one-dimensional heat propagation must be fulfilled; that means that the diameter of the heating beam is wide compared with the thermal diffusion length. Finally, the thermal parameters of the bulk material must be known. Now, an expression for the surface temperature can be derived whereas the depth dependent property $k$ is approximated by a piecewise linear function. Such a polygon curve represents a sufficient approximation for any real profile and a solution of the HDE can be used to describe the thermal waves within each sublayer [6].

In addition, numerical methods like the Finite-Element-Method (FEM) and the FiniteDifference-Method (FDM) can be used $[10,11]$. The FEM, well known from mechanical applications, offers a very comfortable possibility to receive the temperature distribution within the whole sample. For faster calculations, the FDM seems to be a better tool. Herein, the reduced thermal diffusion equation becomes an ordinary differential equation with a corresponding boundary value problem. For a numerical simulation the differential quotients, the derivatives of the temperature $T$, are replaced by discrete difference quotients. Since the temperature at any given point inside the sample is a function of its neighbours, an equation array must be solved for the complete description of the temperature distribution. For a given profile of thermal parameters, including the thermal conductivity, the specific heat and the density, a calculation of the temperature distribution is possible - even in cases where an analytical solution is not available. Or vice versa, the profile of thermal parameters can be reconstructed from photothermal data. This last task is known as the 'inverse problem', which addresses the difficulty to retrieve inhomogeneous material properties in the subsurface region from the measurement of the temperature distribution at the surface. The inversion itself is performed from the modulation frequency sweeps of the exciting laser beam on any side on the sample's surface.

\section{Experimental results- investigations on ceramic materials}

\subsection{Mechanical load - model of Vickers indentation}

The systematic investigations on Vickers indentations in a polished ceramic sample of $\mathrm{Si}_{3} \mathrm{~N}_{4}$ (WIDIANIT 2000) with different loads (2 kg - 50 kg, HV2 - HV50) has been already presented and discussed in [5]. In order to get information about the size of the disturbed areas an inversion procedure described in [6] was used. The amplitude and phase signals on various loaded areas near and far from the Vickers indented surfaces were measured as a function of the modulation frequeny. The contrasts are shown in figure 3.

Now, knowing the thermal parameters of WIDIANIT $2000\left(\mathrm{k}_{0}=0.29 \mathrm{~W} \mathrm{~cm}^{-1} \mathrm{~K}^{-1}, \rho C=1.46 \mathrm{~J} \mathrm{~K}^{-1}\right.$ $\mathrm{cm}^{-3}$ ) and applying the inversion procedure one is able to reconstruct the depth profile of $\mathrm{k}$ assuming $\rho \mathrm{C}$ being constant. This assumption is reasonable because of the very small compressibility of ceramics. The found thermal conductivity profiles are summarized in figure 4. Obviously, a considerable decrease in $\mathrm{k}$ has been induced by the mechanical load. An increase of the load also increases the depths of reduced thermal conductivity. This may be due to a stress-induced plastic deformation followed by a network of optically invisible microcracks inhibiting the penetration of the optically excited thermal waves. In addition, it is worthwhile to mention that the thickness of the distorted areas, the plastically deformed zones, extend considerably the depths of the indented regions. The near-surface reduction of $k$ is nearly the 
same for all loads applied, whereas the reduced thermal conductivity inside deeper regions depends on the load. This effect might indicate, that the thermal diffusion in deeper layers is obstructed mainly by microcracks while the near-surface region is mainly influenced by pressure densifications or plastic deformations.

\subsection{Laser treated ceramics}

In order to demonstrate that photothermal measurements allow additionally the characterization of laser modified surfaces, several experiments on different ceramics have been carried out. E.g., a Widianit workpiece was irradiated by a pulsed Er:YAG laser. We chose this kind of laser excitation because at ambient conditions each part is covered by a thin layer of water. An Er:YAG laser emits light at $2.94 \mu \mathrm{m}$ and water has a very strong absorption band within this region. Almost all the laser light is absorbed in a very thin layer and followed by a very strong thermal pulse which leads to the surface modifications. Afterwards, the samples were examined by photothermal methods as mentioned before. As illustrated in figure 5 the photothermal phase map reveals a highly inhomogeneous surface. Areas of lowered thermal conductivity are marked by darked spots. Only partly, these inhomogeneities could be seen by optical microscopy, whereas vice versa some visible spots could not be seen in the photothermal image, thus, indicating that not all the optical recognizable variations alter the thermal and the mechanical parameters. Figure 6 shows the phase contrast curves. These curves indicate that the variations of the thermal parameters occur over a deep range within the sample. The phase contrast in the low modulation frequency regime may be interpreted as showing that in this case laser irradiation resulted in an increased subsurface fracture, and that a milder treatment is required for surface improvement. Several other experiments have been carried out on hardmetals. Similar results showed that the depth and the profile of thermal variations due to different loads might be reconstructed from photothermal experiments.

\section{Conclusions}

The reconstruction of thermal conductivity profiles from photothermal amplitude and phase signals has been demonstrated for mechanically loaded and laser thermally treated ceramics. Those results confirmed the assumption of the formation of an extended zone of plastic deformation under load which effectively obstructs the diffusion of heat. The depths of the distorted regions depend on the applied impacts and become photothermally detectable. Unfortunately, until now we were not able to compare these results on ceramics with any kind of destructive investigations. One has to consider that mechanical processing such as cutting or grinding will influence the near-surface microstructure - smearing out the mechanically or thermally induced changes. But altogether, the figures and results above justify the assumption that the physical properties of the ceramic samples have been changed in the surroundings of the Vickers indented or thermally deformed areas. The impacts yield changes of thermal properties and, hence, become detectable by photothermal techniques. Up to now, we can not yet decide if a laser thermal treatment improve the characteristics of ceramics. But altogether, the presented results show the ability of photothermal measurements to reconstruct depth profiles of thermal material parameters and, therefore, prove that photothermal inspection of material changes in surfaces and in near-surface layers provide a suitable method for nondestructive evaluation in manufacturing control.

\section{Acknowledgements}

The authors are indebted to Prof. Dr. H.G.Walther, Dr. U.Seidel and Dipl. Phys. T.T.N. Lan for performing the reconstruction calculations on mechanically treated ceramics.

The investigations have been financially supported by the Federal State of Baden-Württemberg Grant No. II7532.294-4/1. 


\section{REFERENCES}

[1] TÖNSHOFF (H.K.), TRUMPOLD (H.), BRINKSMEIER (E.), WOBKER (H.G.): Evaluation of Surface Layers of Machined Ceramics, Annals of the CIRP 38, 2, (1989), 699-708

[2] GOCH (G.), GEERKENS (J.), REICK (M.), SCHMITZ (B.): Analysis of Surface Layer Variations by Photothermal Means, Journal de Physique IV, C7-4, (1994), 319-322

[3] FRIEDRICH (K.), HAUPT (K.), SEIDEL (U.), WALTHER (H.G.): Definition, resolution and contrast in photothermal imaging, J.Appl.Phys. 72, 8, (1992), 3759-3764

[4] SEIDEL (U.), HAUPT (K.), WALTHER (H.G.): Analysis of the detectability of buried inhomogeneities by means of photothermal microscopy, J.Appl.Phys. 75, 9, (1994), 4396-4401

[5] SCHMITZ (B.), REICK (M.), GOCH (G.), STEINER (R.): Photothermal detection of surface defects and thermal changes in near-surface layers, QIRT 94-Eurotherm Series 42, (1994), 293-297

[6] LAN (T.T.N.), SEIDEL (U.), WALTHER (H.G.): The determination of microstructural depth profiles by means of photothermal measurements: Theory, J.Appl.Phys. 77, (1995), 4739-4745

[7] MALKIN (S.), RITTER (J.E.): Grinding mechanisms and strength degradation for ceramics, Journ. Engineering for Industry 111, (1989), 167-174

[8] JACKSON (W.B.), AMER (N.M.), BOCCARA (A.C.), FOURNIER (D.): Photothermal deflection spectroscopy and detection, Appl. Opt. 20, (1981), 1333-1344

[9] KANSTAD (S.O.), NORDAL (P.E.): Experimental aspects of photothermal radiometry, Can. J. Phys. 64, (1986), 1155-1164

[10] GOCH (G.), REIGL (M.): Application of the Finite-Element-Method and the FiniteDifference-Method to photothermal inspections, J. Appl. Phys. 79 (1996), 9084-9089

[11] REIGL (M.), GOCH (G.): Finite-difference-method for depth-profiling of photothermal measurements, presented on this conference

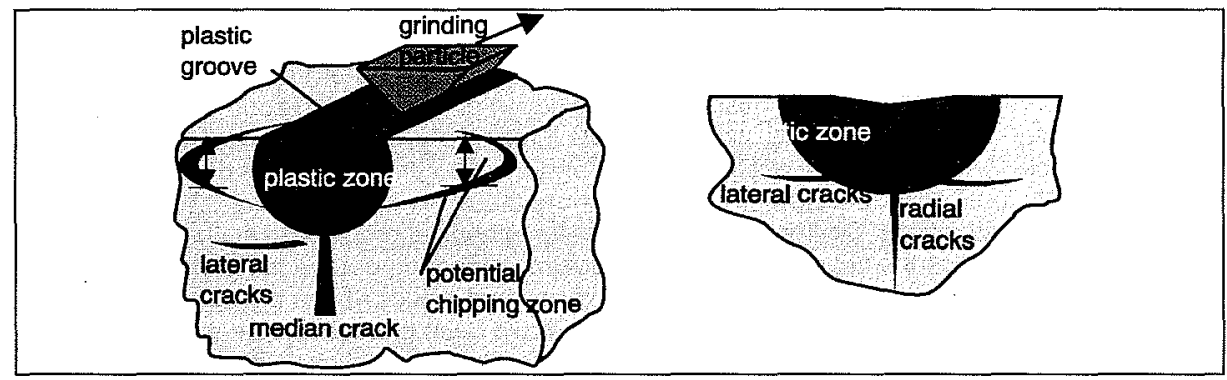

Fig. 1a: Cracks and plastic deformations due to an abrasive diamond grain (acc. to [1])

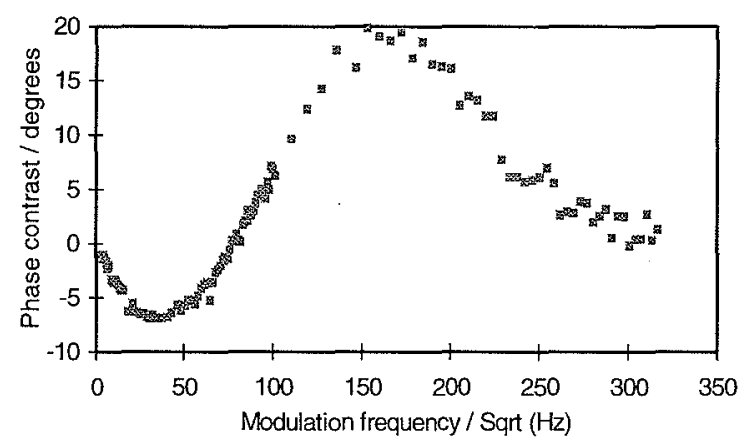

Fig. 6: Frequency sweep on a laser treated ceramic (Widianit), phase contrast 


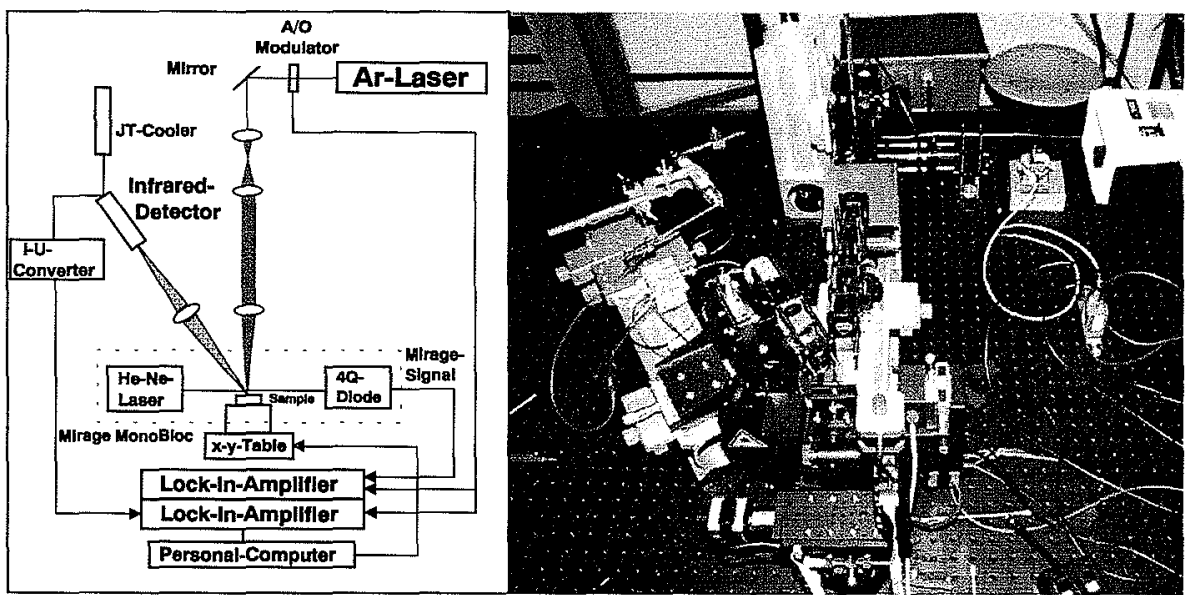

Fig.2: Configuration for photothermal experiments
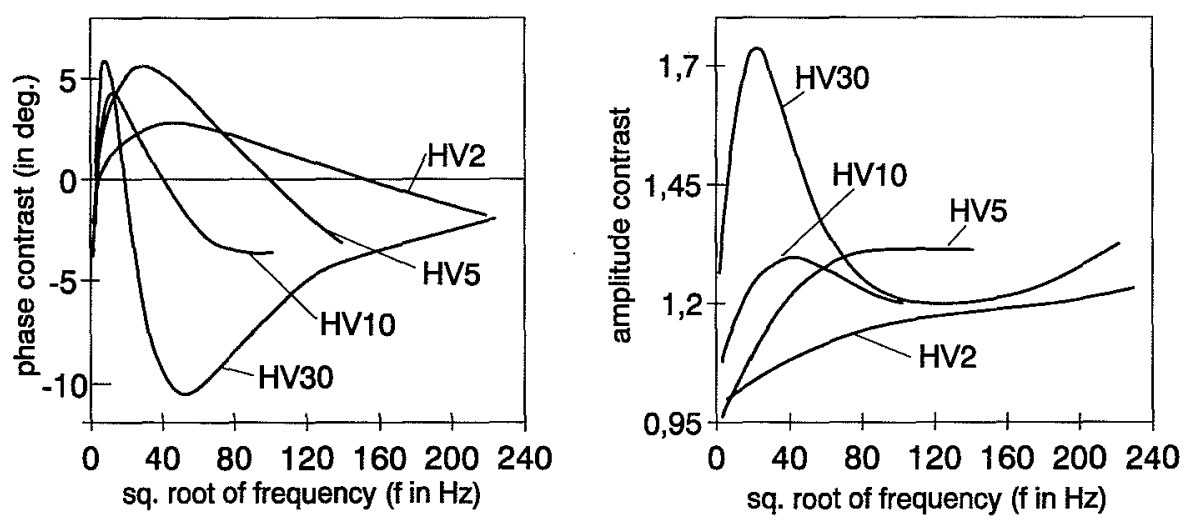

Fig. 3: Phase (left) and amplitude (right) contrasts as functions of the modulation frequency for different loaded Vickers indented areas

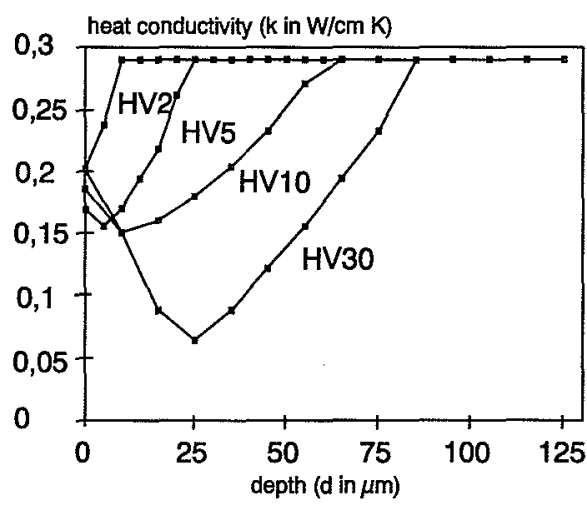

Fig. 4: Reconstructed thermal conductivity profiles of loaded ceramic samples

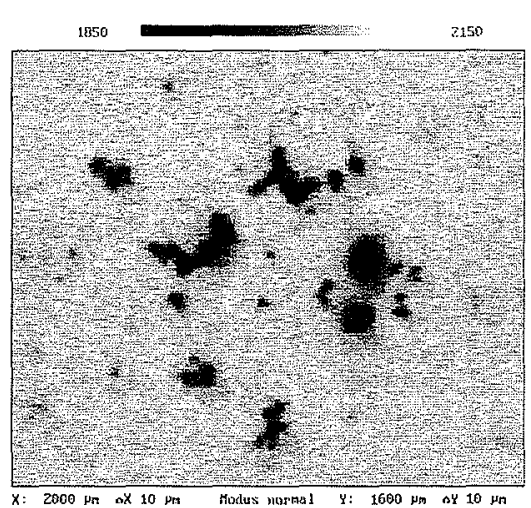

Fig. 5: Photothermal radiometric phase map of a laser treated ceramic surface 